\title{
A Convective Dredge-Up Model as the Origin of Hydrogen in DBA White Dwarfs
}

\author{
B. Rolland, P. Bergeron \& G. Fontaine \\ Département de Physique, Université de Montréal, C.P. 6128, Succ. Centre-Ville, \\ Montréal, Québec H3C 3J\%, Canada \\ rolland@astro.umontreal.ca, bergeron@astro.umontreal.ca, \\ fontaine@astro.umontreal.ca
}

\begin{abstract}
We revisit the problem of the formation of DB white dwarfs, as well as the origin of hydrogen in DBA stars, using a new set of envelope model calculations with stratified and mixed hydrogen/helium compositions. We first describe an approximate model to simulate the so-called convective dilution process, where a thin, superficial hydrogen radiative layer is gradually eroded by the underlying and more massive convective helium envelope, thus transforming a DA white dwarf into a DB star. We show that this convective dilution process is able to account for the large increase in the number of DB white dwarfs below $T_{\text {eff }} \sim 20,000 \mathrm{~K}$, but that the residual hydrogen abundances expected from this process are still orders of magnitude lower than those observed in DBA white dwarfs. Scenarios involving the accretion of hydrogen from the interstellar medium or other external bodies have often been invoked to explain these overabundances of hydrogen. In this paper, we describe a new paradigm where hydrogen, initially diluted within the thick stellar envelope, is still present and slowly diffusing upward in the deeper layers of a $T_{\text {eff }} \sim 20,000 \mathrm{~K}$ white dwarf. When the convective dilution process occurs, the bottom of the mixed $\mathrm{H} / \mathrm{He}$ convection zone sinks deep into the star, resulting in large amounts of hydrogen being dredged-up to the stellar surface, a phenomenon similar to that invoked in the context of DQ white dwarfs.
\end{abstract}

Subject headings: stars: abundances — stars: evolution — stars: fundamental parameters — white dwarfs

\section{INTRODUCTION}

White dwarf stars represent the endpoint of stellar evolution of more than $97 \%$ of the stars in the Galaxy, and the determination of their properties thus provides a variety of 
useful information on the star formation history in our galaxy, and on stellar evolution in general. Having exhausted the nuclear fuel at their center, white dwarfs enter the cooling sequence at effective temperatures around $150,000 \mathrm{~K}$, and gradually cool off at an almost constant radius over several billion years. Because of their high surface gravity of the order of $\log g=8$, chemical separation induced by gravitational settling is quite efficient, and the leftover from previous evolutionary phases are expected to rapidly float to the surface, while heavier elements sink out of sight, thus producing mostly hydrogen- or helium-dominated atmospheres (Schatzman 1958; Paquette et al. 1986). White dwarfs are found in a variety of flavors: The DA stars, whose spectra are dominated by hydrogen lines, comprise about $80 \%$ of the white dwarf population, and their atmospheres are presumably hydrogen-rich. The remaining $20 \%$ are generally referred to as non-DA stars. These include the DB (DO) stars, whose spectra are dominated by neutral (ionized) helium lines, the DQ stars that show molecular carbon Swan bands, the DZ stars with metallic absorption features, and the DC stars, which are completely featureless (Wesemael et al. 1993). Moreover, additional traces of chemical elements are also found at the surface of many white dwarfs, producing a large variety of spectral subtypes (e.g., DAB, DAZ, DBA, DBQ, DBZ, DZA, etc.) depending on the dominant chemical atmospheric constituent. However, these various spectral types are found only in some specific range of temperatures, indicating that there exist several physical mechanisms that compete with gravitational settling to alter the chemical composition of the outer layers of white dwarfs as they evolve along the cooling sequence. Such physical mechanisms include convective mixing, convective dredge-up from the core, accretion from the interstellar medium or circumstellar material, radiative acceleration, and stellar winds (Fontaine \& Wesemael 1987).

Perhaps the most significant signature of this spectral evolution of white dwarf stars is the transformation of DA stars into DB stars below $T_{\text {eff }} \sim 30,000 \mathrm{~K}$ or so. More than 30 years ago, the ultraviolet-excess Palomar-Green (PG) survey (Green et al. 1986) revealed an almost complete absence of helium-atmosphere white dwarfs between $T_{\text {eff }} \sim 45,000 \mathrm{~K}$, where the coolest DO stars were found, and $\sim 30,000 \mathrm{~K}$, where the hottest DB white dwarfs appeared in large number (see also Liebert et al. 1986). This so-called DB gap suggested that a fraction of DA stars, about 20\%, must turn into DB white dwarfs at some point along the cooling sequence. Even though this gap has now been partially filled by the discovery of very hot DB stars in the Sloan Digital Sky Survey (SDSS, Eisenstein et al. 2006), the number of DB stars in the gap still remains a factor of 2.5 lower than what is expected from the luminosity function. The physical mechanism proposed to explain this DB deficiency as well as the sudden increase in the number of DB stars is the float-up model (Fontaine \& Wesemael 1987), where small amounts of hydrogen thoroughly diluted in the envelope of hot white dwarf progenitors, slowly diffuse to the surface, thus gradually 
transforming a helium-dominated atmosphere into a hydrogen-rich atmosphere by the time the white dwarf reaches the blue edge of the DB gap near $T_{\text {eff }} \sim 45,000 \mathrm{~K}$. At lower effective temperatures, $T_{\text {eff }} \lesssim 30,000 \mathrm{~K}$, the onset of the helium convection zone would eventually dilute the superficial radiative hydrogen layer - a process referred to as convective dilution - thus transforming a DA star into a DB white dwarf, provided that the hydrogen layer is thin enough $\left(\log M_{\mathrm{H}} / M_{\odot} \sim-15\right)$.

A second significant signature of the spectral evolution of white dwarf stars occurs below $T_{\text {eff }} \sim 12,000 \mathrm{~K}$ or so, where the fraction of non-DA to DA white dwarfs increases drastically (Greenstein 1986; Tremblay \& Bergeron 2008; Limoges et al. 2015). The most likely explanation for this phenomenon is a process referred to as convective mixing, where the bottom of the superficial convective hydrogen layer plunges into the star as it cools off, eventually reaching the deeper and much more massive convective helium layer if the hydrogen layer is thinner than $\log M_{\mathrm{H}} / M_{\star} \lesssim-6$ Koester 1976; Vauclair \& Reisse 1977; Dantona \& Mazzitelli 1979). At this particular point, hydrogen and helium are convectively mixed into a single mixed $\mathrm{H} / \mathrm{He}$ convection zone. Because the mixing temperature is a function of the mass of the hydrogen layer (for thicker hydrogen layers, the mixing occurs at lower effective temperatures), the resulting hydrogen-to-helium abundance ratio upon mixing is also a function of the thickness of the hydrogen layer.

Both the convective dilution and the convective mixing processes have been explored quantitatively by MacDonald \& Vennes (1991), and more recently by Rolland et al. (2018, hereafter RBF18). In particular, RBF18 showed (see their Figure 16) that the convective mixing scenario predicts $\mathrm{H} / \mathrm{He}$ abundance ratios upon mixing that agree extremely well with those determined in cool $\left(T_{\text {eff }}<12,000 \mathrm{~K}\right)$, He-rich DA white dwarfs where hydrogen (mostly $\mathrm{H} \alpha$ ) is detected. Also shown is that these He-rich DA stars will rapidly turn into DC white dwarfs once hydrogen falls below the limit of detectability. RBF18 also explored quantitatively the outcome of the convective dilution process, by predicting the $\mathrm{H} / \mathrm{He}$ abundance ratios as a function of effective temperature for various thicknesses of the hydrogen layer (see their Figure 14), assuming the dilution process has already occurred. The results indicate that hydrogen layer masses between $\log M_{\mathrm{H}} / M_{\odot}=-13$ and -10 (according to ML2 $/ \alpha=2$ models) are required to account for the presence of hydrogen in the bulk of DBA stars in the range $20,000 \mathrm{~K} \lesssim T_{\text {eff }} \lesssim 12,000 \mathrm{~K}$, analyzed by RBF18. However, white dwarfs with such large hydrogen layer mass would not mix until they reach temperatures below $T_{\text {eff }} \sim 12,000 \mathrm{~K}$ or so. For the convective dilution process to occur, the hydrogen layer mass must be much smaller, of the order of $\log M_{\mathrm{H}} / M_{\odot} \lesssim-15$. For such thin hydrogen layers, the $\mathrm{H} / \mathrm{He}$ ratios predicted in the temperature range where most DBA stars are found are at least three orders of magnitude smaller that the observed ratios. In other words, there is much more hydrogen in the outer layers of DBA stars than expected from 
a simple convective dilution process. This problem has been known for a long time (see MacDonald \& Vennes 1991 and references therein).

Even though there is little doubt that the convective dilution process is responsible for transforming a significant fraction $(\sim 20 \%)$ of DA stars into DB white dwarfs, the origin of hydrogen in DBA stars is still a subject of debate. Since the hydrogen abundances measured in DBA stars appear too high to have a residual origin, external sources of hydrogen have often been invoked to account for the observed abundances, either from the interstellar medium or from other bodies such as comets, disrupted asteroids, small planets, etc. (MacDonald \& Vennes 1991, Jura 2003, Veras et al. 2014, Raddi et al. 2015, Gentile Fusillo et al. 2017). On the other hand, Bergeron et al. (2011) noticed that a lot of the DB white dwarfs in their sample showed no traces of hydrogen, particularly at low effective temperatures, with very stringent upper limits. Such cool hydrogen-deficient DB stars could only have evolved from hotter white dwarf progenitors that contain negligible amounts of hydrogen in their outer stellar envelopes. Based on this observation, Bergeron et al. (2011) found it difficult to reconcile the existence of these "pure" DB white dwarfs with a scenario involving any form of accretion of hydrogen to explain its presence in the photosphere of DBA stars. The authors thus concluded that the presence of hydrogen in DBA stars must be residual, a conclusion also reached by Koester \& Kepler (2015). Bergeron et al. (2011) also suggested that perhaps the convective dilution process is not complete, and that hydrogen somehow floats on top of the photosphere rather than being forcefully mixed by the helium convection zone, although Koester \& Kepler (2015) considered this scenario unlikely since the convection velocities in the mixed $\mathrm{H} / \mathrm{He}$ convection zone are many orders of magnitude larger than the diffusion velocities of hydrogen in helium.

At the same time, some white dwarfs with helium-dominated atmospheres have such exceptionally large hydrogen abundances — which are also correlated with the presence of metals in large quantities - that there is absolutely no doubt that in these cases hydrogen must have been accreted, most likely from water-rich asteroid debris (Farihi et al. 2013; Raddi et al. 2015, Gentile Fusillo et al. 2017 and references therein). Such objects include SDSS J124231.07+522626.6, GD 16, PG 1225-079, GD 362, and GD 17, also reproduced in Figure 16 of RBF18, which shows the exceptionally large hydrogen abundances measured in these stars with respect to other DBA stars and cool, helium-rich DA white dwarfs. More importantly in the present context, Gentile Fusillo et al. (2017) looked at the population of He-atmosphere white dwarfs from Koester \& Kepler (2015), and found that the incidence of hydrogen was strongly correlated with the presence of metals, suggesting that perhaps the majority of helium-rich atmosphere white dwarfs containing hydrogen and metals are likely to have accreted at least some fraction of their hydrogen content in the form of water or hydrated minerals in rocky planetesimal. Since hydrogen will always remain in the mixed 
$\mathrm{H} / \mathrm{He}$ convection zone, while the metals will eventually diffuse away from the photospheric regions, perhaps this accretion mechanism can explain the presence of hydrogen in all $D B A$ stars.

The question of the accretion of hydrogen, either from the interstellar medium or from other external bodies, has been investigated by RBF18 - see their Section 4.4 and Figure 15. By assuming an average accretion rate over the years, RBF18 found that the required amount of accreted material, with even a moderate accretion rate, would build a superficial hydrogen layer thick enough by the time the white dwarf reaches a temperature of $T_{\text {eff }} \sim 30,000 \mathrm{~K}$, that this object - presumably a DA star — would never turn into a helium-atmosphere DB white dwarf. Based on these arguments, it was concluded that the hydrogen abundances measured in DBA stars could not be accounted for by any kind of accretion mechanism onto a pure helium DB star progenitor. Of course, one obvious solution around this problem is to invoke a scenario where accretion of hydrogen-rich asteroid debris begins only after the DA-to-DB transition has occurred, in which case accretion would proceed on a white dwarf with an atmosphere already convectively mixed. Note that so far, this is the only viable scenario to account for the large hydrogen abundances measured in the bulk of DBA white dwarfs. But the question remains: even though there is probably no doubt that hydrogen is accreted in large amounts in some helium-rich white dwarfs, is accretion responsible for the presence of hydrogen in the bulk of DBA stars?

Given the importance of this question, and given the fact that RBF18 used a rather crude envelope models in their exploratory calculations, we first describe in Section 2 improved models that allow us to simulate the convective dilution process in greater detail. Our simulations of convective dilution and of accretion onto DB white dwarfs based on these more realistic models are presented in Section 3. We find similar conclusions as RBF18 at the qualitative level. We next present in Section 4 an alternative scenario where hydrogen might be dredged up from the deep envelope interior. This is based on the general observation that large amounts of hydrogen (compared to what is needed to form a DA atmosphere, $\sim 10^{-15}$ $\left.M_{\odot}\right)$ might still be diluted in the deep envelope, especially if a typical DBA white dwarf

descend from PG1159 objects as discussed, for instance, in Quirion et al. (2012). We further discuss our results and conclude in Section 5 .

\section{The Convective Dilution Process}

As discussed above, RBF18 showed in their Figure 14 the outcome of the convective dilution process - i.e. the $\mathrm{H} / \mathrm{He}$ abundance ratios predicted at the photosphere as a function of effective temperature for various thicknesses of the hydrogen layer — by assuming the 
dilution process has already occurred. In other words, the convective dilution process, per say, was not modeled in any way. MacDonald \& Vennes (1991) used a similar approach, and jumped discontinuously from hydrogen-rich to helium-rich envelope models (see the case with $M_{\mathrm{H}}=10^{-14} M_{\odot}$ in their Figure 1) to describe what these authors call the convective dredge-up process (see their Table 1).

RBF18 explored the convective dilution scenario by using two sets of envelope model calculations: models with homogeneous $\mathrm{H} / \mathrm{He}$ abundance profiles, and chemically stratified models. In the case of stratified models, the hydrogen layer of a given mass was forced to sit on top of the helium layer, in a very approximate way, and was thus never allowed to mix with the underlying helium envelope. This is obviously not a physically realistic situation, in most cases. A more suitable and physical approach is thus required to model properly the kind of stellar envelopes associated with the convective dilution scenario. We describe a new approach in this section, which allows us to follow the convective dilution process in greater detail, and in particular to predict the mixing temperatures more accurately. The reader interested only in the results may skip the remainder of this section and go directly to Section 3 where the most relevant results are presented.

\subsection{Model Envelope Structures}

To explore further the convective dilution process, we use the latest version of the Montréal white dwarf model-building code, which includes the same input physics as the full evolutionary models described at length in Fontaine et al. (2001), with updates discussed in Van Grootel et al. (2013). Here we take advantage of a customized version of the code in its envelope configuration (see Brassard \& Fontaine 1994 for a first description) where an arbitrary abundance profile can be provided as an input to the model in the form of the mass fractions of hydrogen, helium, carbon, and oxygen as a function of depth. If possible, the program will then try to compute a self-consistent numerical solution with these fixed prerequisites. We use this particular feature of our code to revise the stratified and homogeneous models of RBF18, which we describe in turn.

In RBF18, our stratified models were built using a very rough approximation, where the hydrogen layer was basically sitting on top of the helium envelope, leading to spurious convection zones extending over a few layers near the $\mathrm{H} / \mathrm{He}$ transition region of our hottest models (see Figure 11 of RBF18). These where due to too sharp a transition in composition at the $\mathrm{H} / \mathrm{He}$ interface. These approximate thermodynamic structures will be referred to as seed models for our improved calculations. Here, we thus go one step further and first solve the diffusion equation to compute the equilibrium $\mathrm{H} / \mathrm{He}$ abundance profile (see, e.g., 
Vennes et al. 1988) using these approximate seed models. The resulting abundance profile is then fed back into our code to recompute a new thermodynamic structure that is consistent with this profile; the entire procedure is repeated until convergence. The hydrogen mass fraction as a function of depth resulting from this improved model structure is compared in Figure 1 (red solid line) with our previous calculations taken from RBF18 (red dashed line) for a typical $0.6 M_{\odot}$ model at $T_{\text {eff }}=18,000 \mathrm{~K}$ with $\log M_{\mathrm{H}} / M_{\star}=-13.77\left(M_{\star}\right.$ is the mass of the star). As can be seen, the $\mathrm{H} / \mathrm{He}$ abundance profile in diffusive equilibrium from our improved calculations provides a much smoother transition between the hydrogen and helium layers than our previous models where the transition region is fairly abrupt.

The effect of these improved calculations on the extent of the convection zones, as a function of $T_{\text {eff }}$, is illustrated in Figure 2 for models at $0.6 M_{\odot}$ with $\log M_{\mathrm{H}} / M_{\star}=-13.77$, where our new results are compared with our earlier calculations presented in RBF18 (see their Figure 11). The spurious convection zones near the $\mathrm{H} / \mathrm{He}$ transition region that were present in our earlier calculations (see the discussion above) have all but vanished in our improved models. Also, we can see that at lower effective temperatures, the extent of the helium convective zone has slightly shifted upwards. Note that in our calculations, we neglect the fact that the abundance profile should be constant throughout the convection zone (as opposed to what is displayed by the red solid line in Figure 1), because in all cases explored here, either the extent of the convection zone is extremely small — as in Figure 2 - or hydrogen remains a trace element, and is thus not affecting the extent of the convection zone. For completeness, we would like to point out that the models displayed in Figure 2 are comparable to the solution A shown in Figure 6 of MacDonald \& Vennes (1991), where some small amounts of hydrogen is mixed within a shallow helium convection zone, but where most of the hydrogen floats at the surface of the star.

Note that at some effective temperature and for some thickness of the hydrogen layer, the deep helium convection zone will become so efficient as to dilute the superficical hydrogen layer, and the stratified envelope structures described above will no longer be appropriate. The fundamental problem is to determine at what temperature and for which value of the hydrogen layer mass will this dilution process occur. To answer these questions we develop a new set of hybrid envelope structures which we now describe.

We thus revisited our chemically homogeneous models from RBF18 (see their Figures 9 and 10), in which a constant and homogeneous $\mathrm{H} / \mathrm{He}$ composition was assumed from the surface to the bottom of the stellar envelope, characterized by $M_{\text {env }} / M_{\star}=10^{-2}$. These earlier models will now serve as seed models for our improved hybrid envelope calculations. Here the constant $\mathrm{H} / \mathrm{He}$ abundance ratio (or equivalently, the hydrogen mass fraction) throughout the stellar envelope is replaced by a piece-wise function that follows these simple criteria: (1) the 


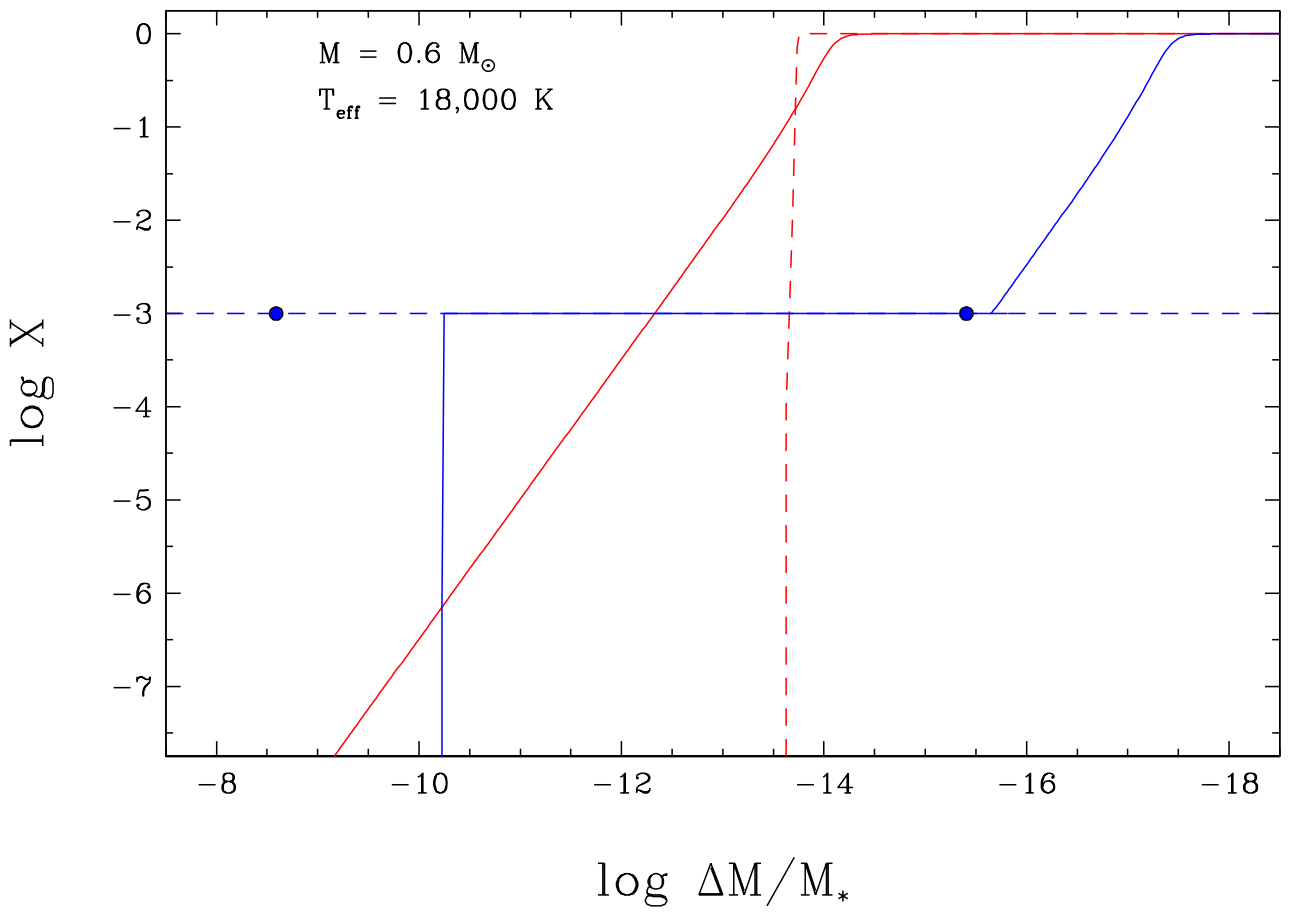

Fig. 1.- Hydrogen mass fraction as a function of depth, expressed as the fractional mass above the point of interest with respect to the total mass of the star, for various types of envelope structures at $T_{\text {eff }}=18,000 \mathrm{~K}$. Chemically stratified $\left(\log M_{\mathrm{H}} / M_{\star}=-13.77\right)$ and mixed $\mathrm{H} / \mathrm{He}$ models $(\log X=-3)$ are shown in red and blue, respectively. Our approximate seed models are represented by dashed lines, while our improved stratified and hybrid envelope structures are shown as solid lines (see text). The extent of the convection zone in the seed mixed model is indicated with blue dots. All models have been calculated with the ML2 $/ \alpha=0.6$ parameterization of the mixing-length theory. 


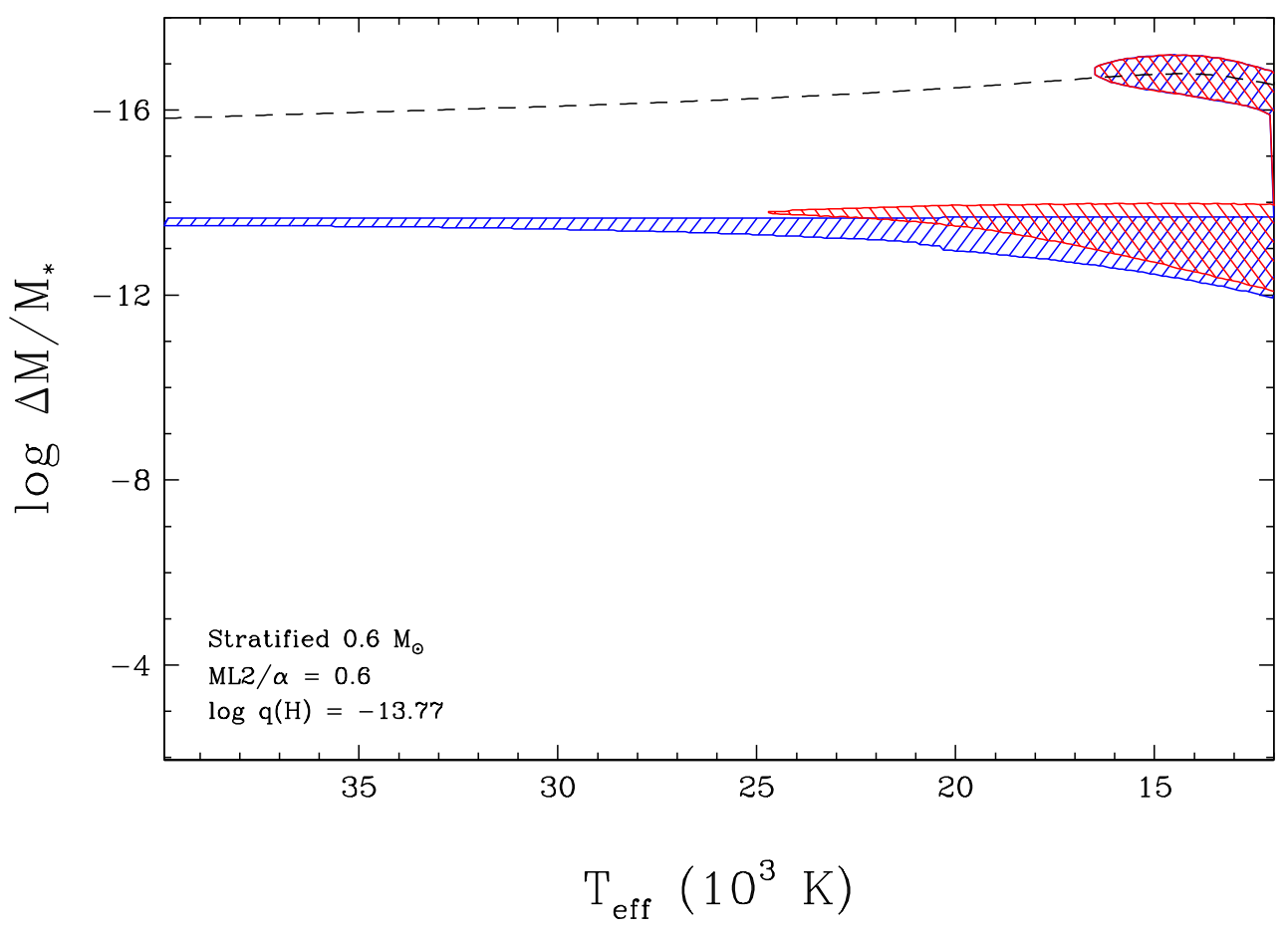

Fig. 2.- Examples of envelope structures for a $0.6 M_{\odot}$ white dwarf with chemically stratified compositions. The extent of the convection zones are shown by the hatched regions. The models shown in blue correspond to the original calculations presented in RBF18, while those in red are obtained by solving the diffusion equation to compute the equilibrium $\mathrm{H} / \mathrm{He}$ abundance profile (see also Figure 1). The dashed line indicates the location of the photosphere in both models. 
$\mathrm{H} / \mathrm{He}$ abundance profile above the convection zone is assumed to be in diffusive equilibrium 1 ; (2) the abundance profile must be continuous from the bottom of the convection zone to the surface; (3) the abundance profile is assumed to be constant throughout the convection zone, and set to the $\mathrm{H} / \mathrm{He}$ abundance ratio of the corresponding seed model; (4) the hydrogen abundance below the convection zone is set to zero. As discussed in RBF18, the presence of hydrogen below the convective layer has no effect on the extent of the convection zone.

An example of this hybrid construct is displayed in Figure 1 for a $0.6 M_{\odot}$ model at $T_{\text {eff }}=18,000 \mathrm{~K}$ (blue solid line); the constant hydrogen mass fraction of the corresponding seed model is also indicated (blue dashed line). Contrary to our previous calculations, the hydrogen abundance above the convection zone (the convection zone is represented by the flat region at $\log X=-3$ ) increases steadily towards the surface, as expected from a diffusive equilibrium profile (see also Figure 6 of MacDonald \& Vennes 1991). More importantly in the present context, the presence of hydrogen at the surface has the effect of modifying the extent of the mixed $\mathrm{H} / \mathrm{He}$ convection zone underneath, as indicated by the two blue dots in Figure 1, which show the extent of the convection zone in our homogeneous seed model. In particular, the bottom of the convection zone has moved significantly higher in the envelope of our new models. Eventually, if there is too much hydrogen at the surface, convection in the deeper envelope will become completely inhibited. When this situation occurs, we should recover the chemically stratified models described above. This will become the branching point of the convective dilution process described in Section 2.3. Again, we would like to point out that our improved hydrid model displayed in Figure 1 is comparable, in this case, to the solution E shown in Figure 6 of MacDonald \& Vennes (1991), where a large amount of hydrogen is mixed within a deep helium convection zone, with very little hydrogen floating at the surface of the star.

Following this procedure, we replaced our original grid of homogeneous models with a full grid of these hybrid envelope structures assuming both the ML2/ $\alpha=0.6$ and $\alpha=2.0$ parameterizations of the mixing-length theory to treat convective energy transport. As discussed in RBF18, these two values bracket the convective efficiencies mostly used in the context of white dwarf atmospheres and envelopes. Our independent variable for the construction of these hybrid models is the hydrogen mass fraction in the convection zone, labeled $X_{\mathrm{c} d} 2$. Our grid covers a range of effective temperature between $T_{\text {eff }}=12,000 \mathrm{~K}$ and $60,000 \mathrm{~K}$ by steps of $100 \mathrm{~K}$, and $\log X_{\mathrm{cz}}=-7.75$ to -0.25 by steps of 0.25 dex, thus covering

\footnotetext{
${ }^{1}$ This approximation is identical to that described in MacDonald \& Vennes (1991).

${ }^{2}$ If more than one convection zone is present, the hydrogen mass fraction of the deepest and most massive convection zone - usually associated with He II — is adopted.
} 
essentially the entire range between pure helium and nearly pure hydrogen compositions.

The hydrogen mass fraction as a function of depth is displayed in Figure 3 for $0.6 M_{\odot}$ models at $T_{\text {eff }}=20,000 \mathrm{~K}$, and with various values of the hydrogen mass fraction in the mixed $\mathrm{H} / \mathrm{He}$ convection zone $\left(X_{\mathrm{cz}}\right)$. In each model, the extent of the convection zone can be identified by the flat part of the abundance profile. Note that, because of the way these models are constructed, the total hydrogen mass present in the stellar envelope varies as a function of effective temperature for a given value of $X_{\mathrm{cz}}$.

\subsection{Total Hydrogen Mass}

The new calculations described in the previous section allow us to explore the convective dilution process in greater detail. We remind the reader that this process describes the outcome of a DA white dwarf for which the superficial hydrogen layer has been thoroughly diluted within the underlying helium convection zone. To predict the behavior of this physical process as a function of time (i.e., with decreasing effective temperature), we must perform a detailed analysis of the $T_{\text {eff }}-M_{\mathrm{H}}$ parameter space in order to identify the contours of constant total hydrogen mass. To do so, we first compute the total amount of hydrogen present in each stellar envelope of our hybrid model grid. The results of these calculations are presented in Figure 4 where we show the total hydrogen mass content, $M_{\mathrm{H}}$, as a function effective temperature for various values of the hydrogen mass fraction in the convection zone, $X_{\mathrm{cz}}$.

The first feature that arises naturally from our calculations is the existence of an upper limit to the total hydrogen mass that a white dwarf can contain given our assumed envelope structures. For instance, there is no envelope structure above $T_{\text {eff }} \sim 22,000 \mathrm{~K}$ with a total hydrogen mass of $\log M_{\mathrm{H}} / M_{\odot}=-14.5$ (for ML2/ $\alpha=0.6$ models). Stellar envelopes having such a large, or even larger, hydrogen mass would require $\log X_{\mathrm{cz}} \rightarrow 0$ (i.e. $X_{\mathrm{cz}} \rightarrow 1$ ), and can only exist if their structure is chemically stratified, corresponding to the DA star configurations described earlier. Since similar constraints can be obtained at all effective temperatures, this defines a region in the $T_{\text {eff }}-M_{\mathrm{H}}$ parameter space, represented by the red hatched area in Figure 4, where white dwarfs can only exist as chemically stratified DA stars.

By using the results displayed in Figure 4, it is now possible to follow the evolution of $X_{\mathrm{cz}}$ as a function of decreasing effective temperature for a given value of $M_{\mathrm{H}}$ by reading the corresponding value of $X_{\mathrm{cz}}$ at each temperature. One can see that there is a unique solution for $X_{\mathrm{cz}}$, at any given effective temperature, for all values of $\log M_{\mathrm{H}} / M_{\odot}<-16$. For larger 


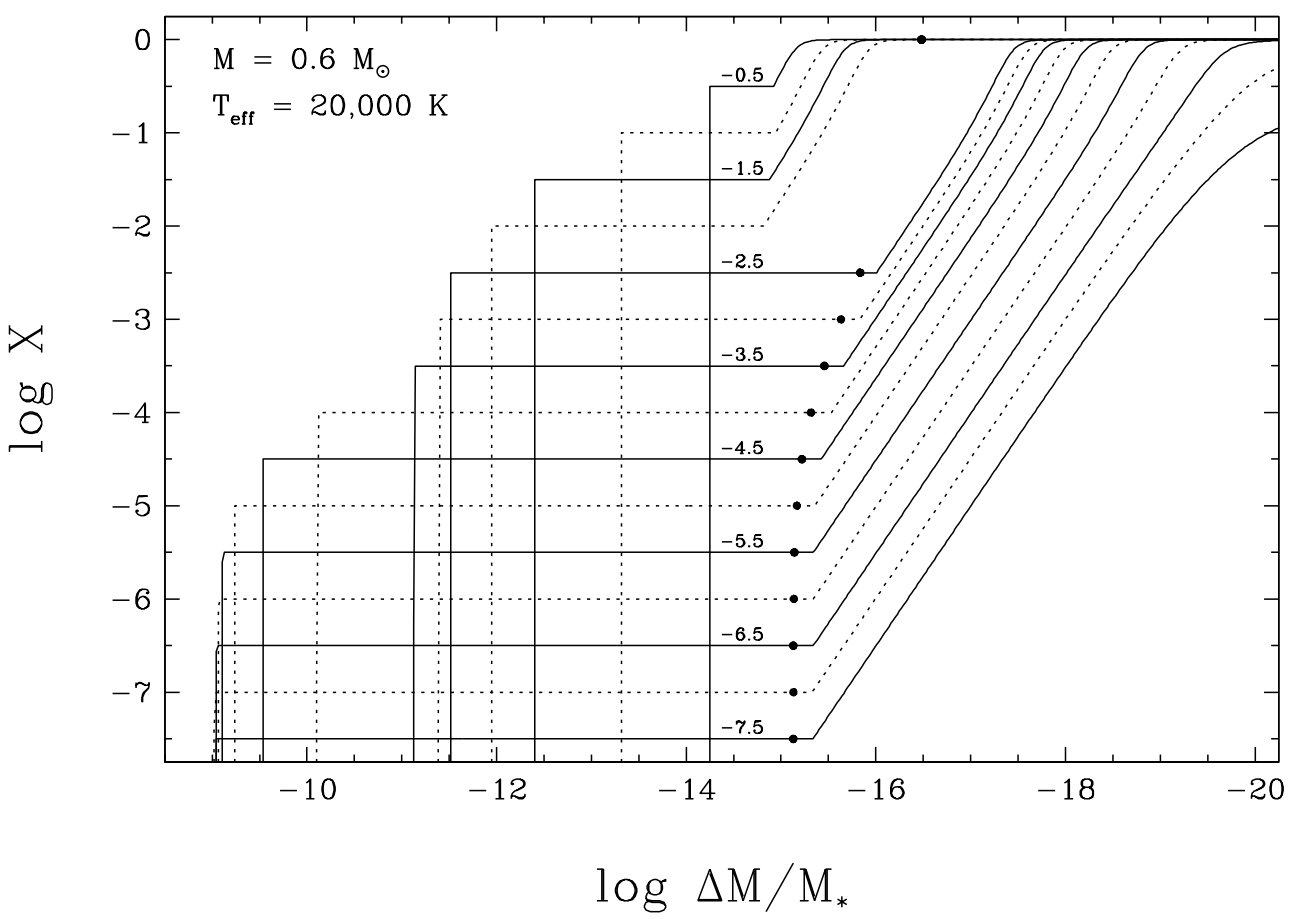

Fig. 3.- Hydrogen mass fraction as a function of depth for our hybrid envelope models at $0.6 M_{\odot}, T_{\text {eff }}=20,000 \mathrm{~K}$, and with various values of the hydrogen mass fraction in the mixed $\mathrm{H} / \mathrm{He}$ convection zone $\left(\log X_{\mathrm{cz}}\right)$, labeled in the figure. The black dots indicate the location of the photosphere in each model. Only the results for ML2/ $\alpha=0.6$ are displayed here. 


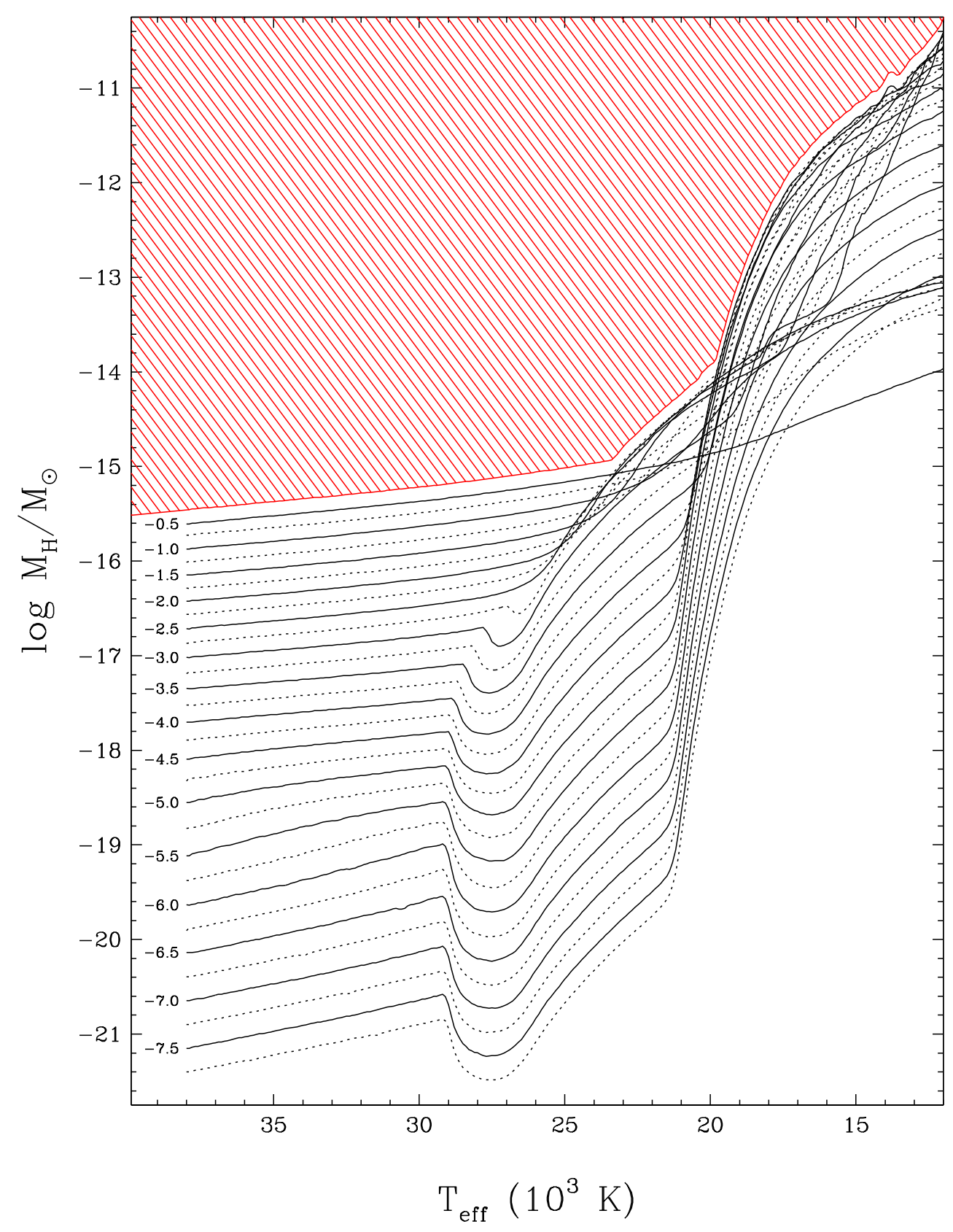

Fig. 4.- Total hydrogen mass, $M_{\mathrm{H}}$, in our hybrid envelope models at $0.6 M_{\odot}$ as a function of effective temperature, and for various values of the hydrogen mass fraction in the convection zone, $X_{\mathrm{cz}}$, labeled on each curve. The region where white dwarfs should appear as DA stars is indicated by the red hatched area. Only the results for ML2 $/ \alpha=0.6$ are displayed here. 
values of $M_{\mathrm{H}}$, but below the red hatched region, there is also a unique solution as long as the temperature remains high $\left(T_{\text {eff }} \gtrsim 25,000 \mathrm{~K}\right)$. At lower temperatures and higher total hydrogen content, however, there are multiple solutions for the same value of $M_{\mathrm{H}}$, generally separated by orders of magnitude. These degeneracies simply reflect the possibility of mixing the same total amount of hydrogen in a deep, or in a shallow, convection zone. Note that MacDonald \& Vennes (1991) also found such multiple solutions for some $T_{\text {eff }}$ values — see in particular their Figures 1 and 6 - some of which were unstable. In general, there is one solution corresponding to a DA star configuration (the chemically stratified model), and one corresponding to a DBA star configuration (hydrogen appears as a trace in the mixed convection zone), but in some cases there is another solution where helium is considered a trace element in diffusive equilibrium within the superficial hydrogen-rich layer (the solution $\mathrm{C}$ in their Figure 6). As discussed by MacDonald \& Vennes, these last solutions probably do not exist in nature. In what follows, we use similar considerations to decide which solution to adopt in our simulations.

\subsection{Convective Dilution Simulations}

As discussed in the previous sections, if there is too much hydrogen at the surface of one of our hybrid model structure, convection in the deeper envelope will become completely inhibited, and at this point we should recover the chemically stratified models described at the beginning of Section 2.1. Thus, in order to follow the spectral evolution of white dwarfs with a constant total hydrogen mass, we start with a chemically stratified model at high effective temperature $\left(T_{\text {eff }} \sim 60,000 \mathrm{~K}\right)$ and a given value of $M_{\mathrm{H}}$. We then search a model within our grid of hybrid envelope structures in the $T_{\text {eff }}-M_{\mathrm{H}}$ parameter space (see Figure 4) for which the shallow convection zone corresponds to its stratified counterpart 3 . If no such convective envelope exists, we repeat the procedure with a slightly cooler stratified model until an appropriate match is found. Since we are interested in DBA star configurations where hydrogen is always a trace element, we adopt in Figure 4 the appropriate solution for $X_{\mathrm{cz}}$ that is representative of DBA white dwarfs. This approach offers a simple and efficient way to discriminate between the different $T_{\text {eff }}-M_{\mathrm{H}}$ degeneracies at a given effective temperature, and thus ensures a near-continuity in the evolution of the thermodynamic structures. From that point on, we only rely on our hybrid envelope structures. The results of our convective dilution simulations are presented in the next section.

\footnotetext{
${ }^{3}$ Note that such shallow convection zones exist even in our chemically stratified structures, as shown in Figure 2.
} 


\section{RESULTS}

Examples of our envelope structures with constant values of the total hydrogen mass present in the stellar envelope, $M_{\mathrm{H}}$, are displayed in Figures 5 and 6 . The location of the photosphere as well as the extent of the convection zones are indicated in each panel. Even though these sequences represent static envelope models, we believe they provide a good representation of the convective dilution scenario, which, in reality, is a much more complex dynamical process. We first note that, within our framework, white dwarfs with a total hydrogen mass of $\log M_{\mathrm{H}} / M_{\odot} \geq-13.75$ will never undergo dilution. Such objects should remain DA stars until the superficial hydrogen convection zone reaches the deeper convective helium layers (when $T_{\text {eff }}<12,000 \mathrm{~K}$ ), at which point convective mixing occurs if the hydrogen layer is not too thick (see Figure 16 of RBF18). At the other end of the mass spectrum, our models are almost identical to pure helium-envelope white dwarfs if $\log M_{\mathrm{H}} \lesssim$ $-16.0 M_{\odot}$. For such thin hydrogen layers, there is not enough hydrogen accumulated at the surface of the star to appear as a DA white dwarf (see Figures 3 and 4 of Manseau et al. 2016). Instead, these objects would appear as stratified DAB stars, with a thin hydrogen layer floating in diffusive equilibrium at the stellar surface.

Our version of the convective dilution mechanism should thus be effective for white dwarf envelopes containing an amount of hydrogen ranging from $10^{-16} M_{\odot}$ to $10^{-14} M_{\odot}$. The transition temperatures at which this physical process is expected to occur are summarized in Table 1 for both the ML2/ $\alpha=0.6$ and $\alpha=2.0$ parameterizations of the mixing-length theory. With ML2/ $\alpha=0.6$ for instance, we can see that depending on the thickness of the hydrogen layer, the DA-to-DB transition takes place over a narrow range of mixing temperatures, namely between $T_{\text {eff }} \sim 25,000 \mathrm{~K}$ and $\sim 14,000 \mathrm{~K}$. The sudden transition in surface abundance can be observed in Figures 5 and 6 when the photosphere, indicated by the red line, moves from the radiative hydrogen layer into the mixed $\mathrm{H} / \mathrm{He}$ convection zone. As noted by many authors, including MacDonald \& Vennes (1991) and RBF18, there is a direct correlation between the mixing temperature and $M_{\mathrm{H}}$, the latter delaying the growth of the underlying helium convection zone. Even if our approach is not based on time-dependent calculations, we can expect the duration of this dynamical transformation to be much shorter than the typical cooling time, spanning at most $500 \mathrm{~K}$, or $\sim 10^{7}$ years. Since the depletion of the superficial hydrogen layer leads to a rapid growth of the helium convection zone, convective dilution can be viewed as a cascade-like process, which becomes increasingly efficient as hydrogen gradually disappears from the surface. 


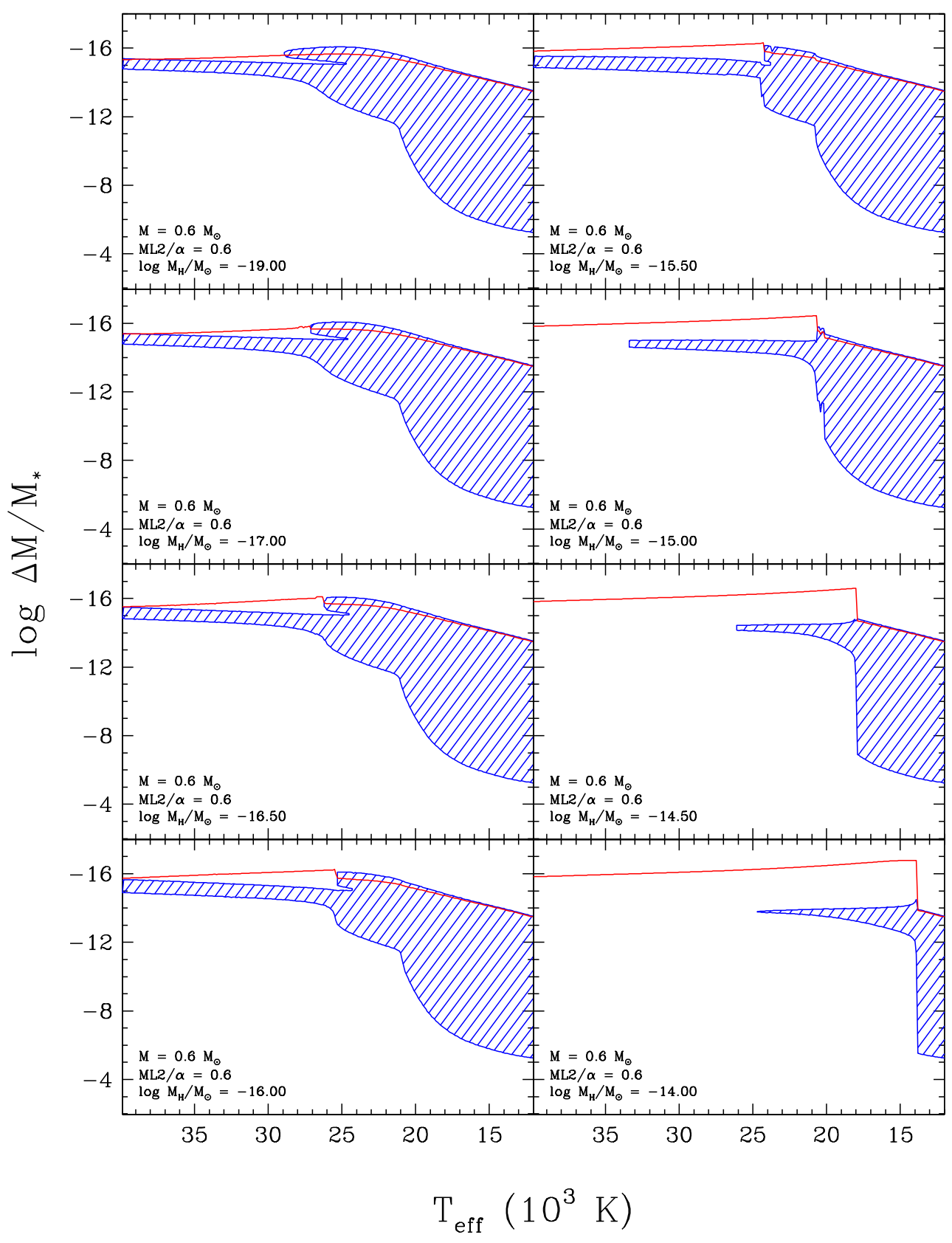

Fig. 5.- Examples of envelope structures for white dwarf models with ML2/ $\alpha=0.6$ subject to a convective dilution process as a function of effective temperature. The depth is expressed as the fractional mass above the point of interest with respect to the total mass of the star. The red solid line corresponds to the location of the photosphere, and the convection zones are shown by the hatched region. The models illustrated here are (from upper left to bottom right) for $0.6 M_{\odot}$ with increasing total hydrogen mass in the stellar envelope. 


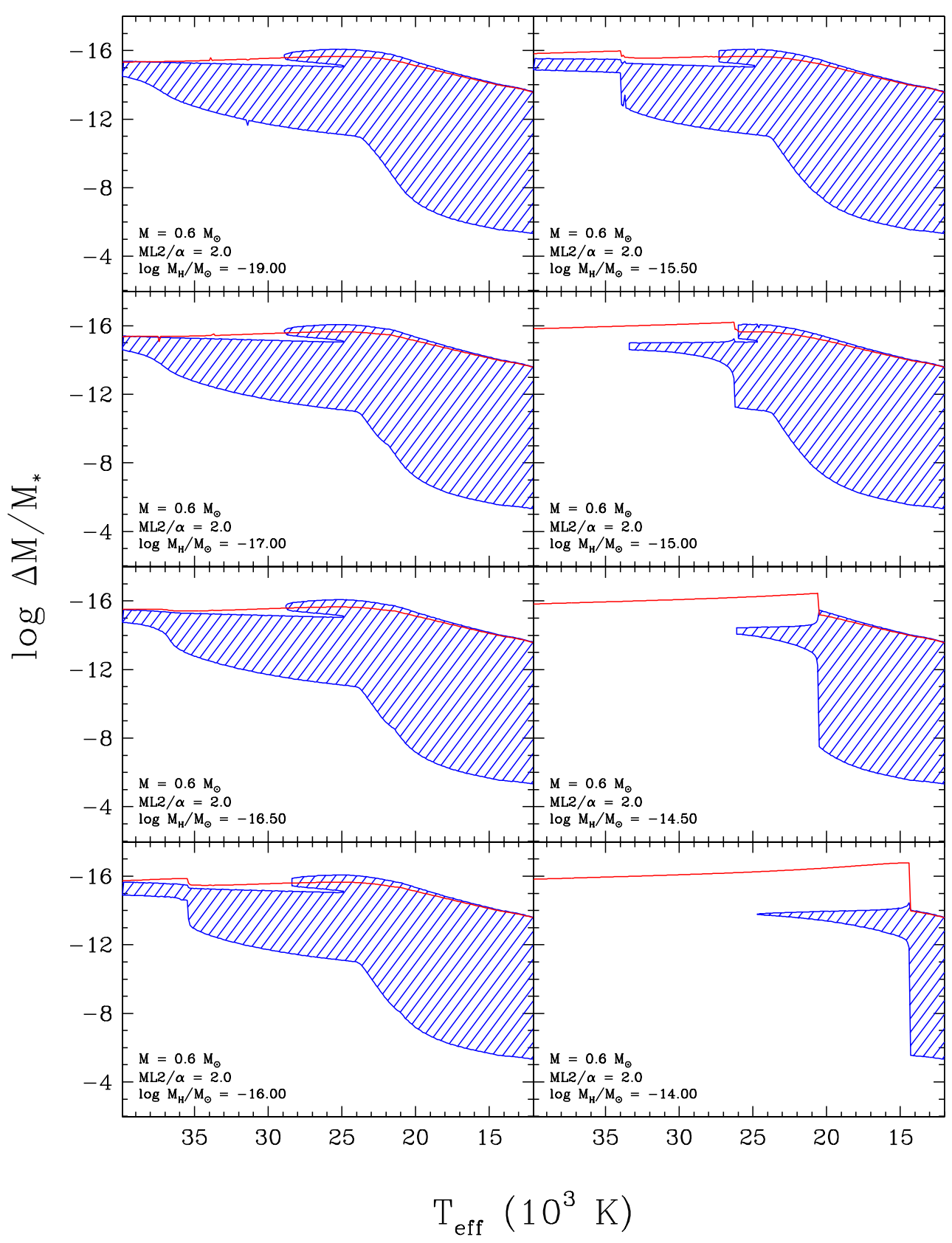

Fig. 6. - Same as Figure 5 but for ML2/ $\alpha=2.0$. 
Table 1. Hydrogen- to Helium-Atmosphere Transition Temperatures

\begin{tabular}{ccrr}
\hline \hline & $\alpha=0.6$ & & $\alpha=2.0$ \\
$\log M_{\mathrm{H}} / M_{\odot}$ & $T_{\text {eff }}(\mathrm{K})$ & & $T_{\text {eff }}(\mathrm{K})$ \\
\hline-16.00 & 25,400 & & 35,400 \\
-15.75 & 24,800 & & 34,500 \\
-15.50 & 24,200 & & 33,900 \\
-15.25 & 23,500 & & 27,200 \\
-15.00 & 20,600 & & 26,200 \\
-14.75 & 19,300 & & 23,600 \\
-14.50 & 17,900 & & 20,500 \\
-14.25 & 16,100 & & 17,300 \\
-14.00 & 13,800 & & 14,300 \\
\hline
\end{tabular}


We now compare the predictions of our convective dilution model with the photospheric $\mathrm{H} /$ He abundance pattern observed in DB and DBA white dwarfs. The results of our simulations are presented in Figure 7, together with the determination of the hydrogen-to-helium abundance ratio, as a function of effective temperature, for all DB and DBA white dwarfs in the sample of RBF18 (see their Figure 5) and in the SDSS sample of Koester \& Kepler (2015). As discussed above, the sudden drops in the predicted $\mathrm{H} / \mathrm{He}$ abundance ratio can be traced back to the results displayed in Figures 5 and 6 when the photosphere moves from the radiative hydrogen layer into the mixed $\mathrm{H} / \mathrm{He}$ convection zone.

Our results indicate that white dwarfs above $T_{\text {eff }} \sim 22,000 \mathrm{~K}$ can easily be explained if the total hydrogen mass is lower than $\log M_{\mathrm{H}} / M_{\odot} \sim-16.5$ with ML2/ $\alpha=0.6$ (or lower than $\log M_{\mathrm{H}} / M_{\odot} \sim-15$ with $\left.\alpha=2.0\right)$. As mentioned above, these objects have never been genuine DA stars at higher temperatures, and their most likely progenitors are the hot DB stars identified in the DB-gap by Eisenstein et al. (2006). Note that the few DBA white dwarfs in this temperature range will rapidly evolve into DB stars, with no detectable traces of hydrogen, as a result of the growth of the helium convective zone with decreasing effective temperature, which should efficiently dilute any residual amount of hydrogen present in the outer layers (see also Koester \& Kepler 2015). Interestingly enough, the DBA star LP 497114 (WD 1311+129) — the most hydrogen-rich object in Figure 7 near $T_{\text {eff }} \sim 22,000 \mathrm{~K}$ - lies close to the $10^{-15.25} M_{\odot}$ transformation branch (for ML2/ $\alpha=0.6$ ), suggesting that this white dwarf might be in the process of being convectively mixed, as suggested by Bergeron et al. (2011, see their Section 5.4), who also reported spectroscopic variations in this object (see their Figure 28).

The bulk of DB and DBA white dwarfs in Figure 7 is found below 20,000 K, however, where the bottom of the helium convection zone sinks rapidly into the stellar envelope. The significant increase in the number of DB white dwarfs in this temperature range can be most easily explained by the convective dilution process, with a total hydrogen mass in the range $-16<\log M_{\mathrm{H}} / M_{\odot}<-14$ according to our calculations. However, if the mixing temperatures predicted by our simulations agree well with the increased number of DB white dwarfs, the amount of residual hydrogen expected after mixing has occurred is several orders of magnitude below the observed abundances in DBA stars, a conclusion also reached in all previous investigations (see RBF18, MacDonald \& Vennes 1991, and references therein). As discussed by RBF18, in order to match the observed hydrogen abundances in DBA white dwarfs, hydrogen layer masses larger than $\log M_{\mathrm{H}} / M_{\odot} \sim-13$ are required, in which case the convective dilution process becomes impossible.

We must therefore conclude that most, but not all, helium-atmosphere white dwarfs cooler than $T_{\text {eff }} \sim 30,000 \mathrm{~K}$ containing traces of hydrogen cannot be explained in terms 


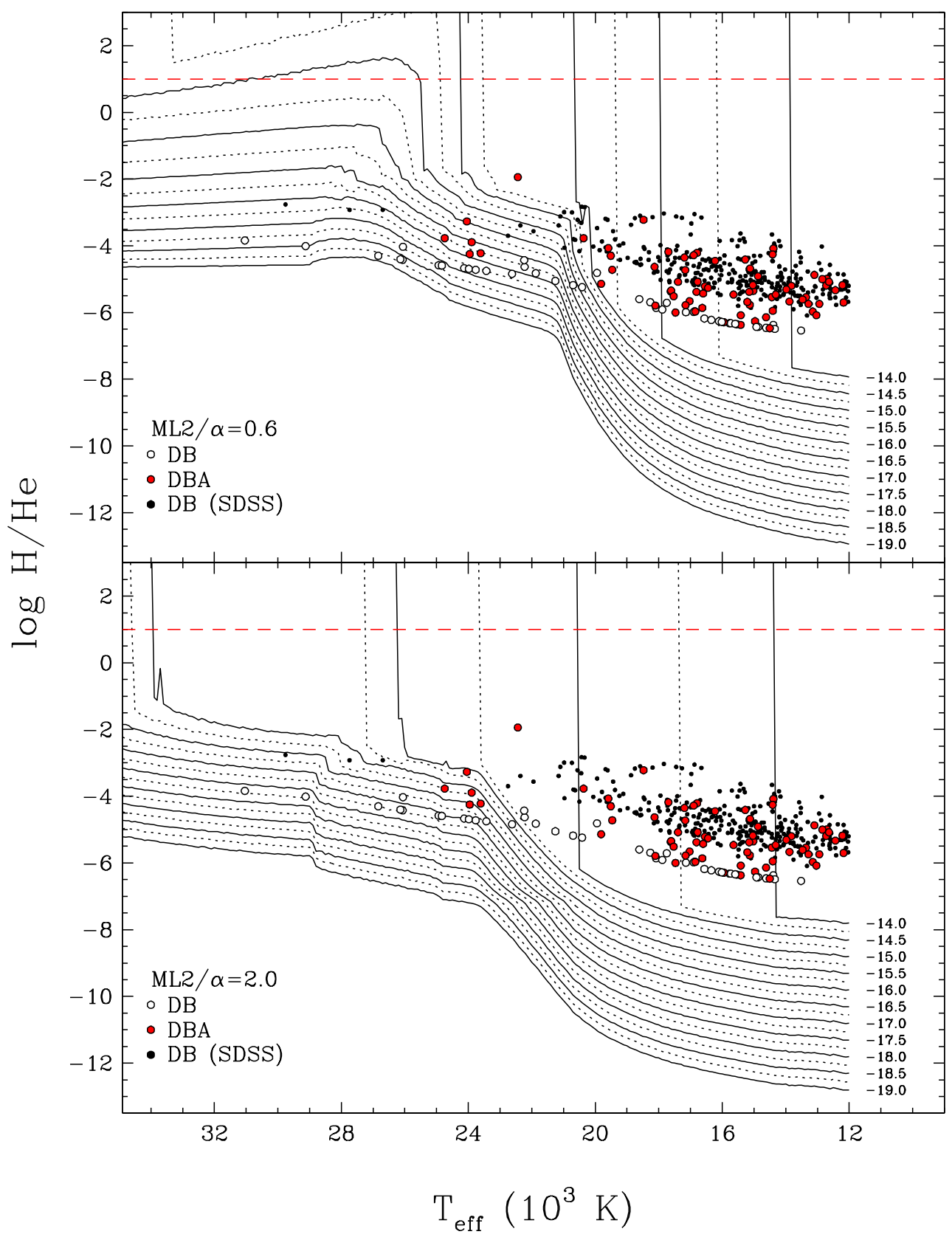

Fig. 7.- Results of our convective dilution simulations for models at $0.6 M_{\odot}$ for the ML2 $/ \alpha=0.6$ and $\alpha=2.0$ versions of the mixing-length theory. Each curve is labeled with the corresponding value of $\log M_{\mathrm{H}} / M_{\odot}$. The red dashed line indicates our empirical limit above which a white dwarf should appear as a DA star. Observed hydrogen abundances, or limits, for DB and DBA white dwarfs from RBF18 and Koester \& Kepler (2015) are also reproduced. 
of our version of the convective dilution scenario. The most common solution proposed to solve this problem is to assume that a significant fraction of DB stars are indeed the result of a convective dilution scenario, with DA progenitors having very thin hydrogen layers $\left(\log M_{\mathrm{H}} / M_{\odot} \leq-15\right)$, but that after the DA-to-DB transition has occurred, accretion of hydrogen from the interstellar medium or other external bodies (comets, disrupted asteroids, etc.) increases the hydrogen content in the stellar envelope, up to the level observed in DBA white dwarfs. But as discussed in RBF18 (see their Figure 15), the amount of accreted material required to account for the observed abundances in DBA stars will most likely build a thick enough hydrogen layer in the earlier evolutionary phases, such that the convective dilution process will again become impossible.

To study the effect of the accretion of hydrogen from external sources more quantitatively, we repeated our previous calculations by considering a constant accretion process onto a pure helium-envelope white dwarf at $0.6 M_{\odot}$. We considered various accretion rates ranging from $\dot{M}=10^{-27} M_{\odot} \mathrm{yr}^{-1}$ to $10^{-16.5} M_{\odot} \mathrm{yr}^{-1}$ by steps of $0.25 \mathrm{dex}$, and calculated the total accreted mass of hydrogen as a function of the white dwarf cooling time, or equivalently, as a function of effective temperature.

The photospheric hydrogen abundances as a function of $T_{\text {eff }}$ predicted by our convective dilution model in the presence of accretion are displayed in Figure 8. These results confirm our previous expectations, that the convective dilution process will occur in the presence of accretion, but only for extremely low accretion rates of $\dot{M} \lesssim 10^{-23} M_{\odot} \mathrm{yr}^{-1}$. This upper limit is 2 to 5 orders of magnitude smaller than the accretion rate required to account for the observed hydrogen abundances in DBA white dwarfs (see Figure 15 of RBF18 in particular). But according to our calculations, such high accretions rates will produce DA star progenitors with hydrogen layers that are thick enough to prevent any form of DA-toDB transformation in the appropriate range of temperature. Note that we exclude here the scenario where large bodies such as small planets, comets, or disrupted asteroids have been accreted after mixing has occurred, as often invoked in objects such as GD 16, GD 17, GD 40, GD 61, G241-6, PG 1225+079, and HS 2253+8023, which all show evidence of accretion of water-rich material (Raddi et al. 2015, Gentile Fusillo et al. 2017). However, we do not believe that this particular scenario applies to the bulk of DBA white dwarfs, although it cannot be completely ruled out.

Hence, we conclude that the amount of hydrogen observed in DBA stars cannot be explained as having a residual origin - the leftovers from the convective dilution scenario - nor can it be accounted for by accretion, at least in terms of an average accretion rate. In the next section, we present an alternative possibility as a solution to the problem of hydrogen in DBA white dwarfs. 


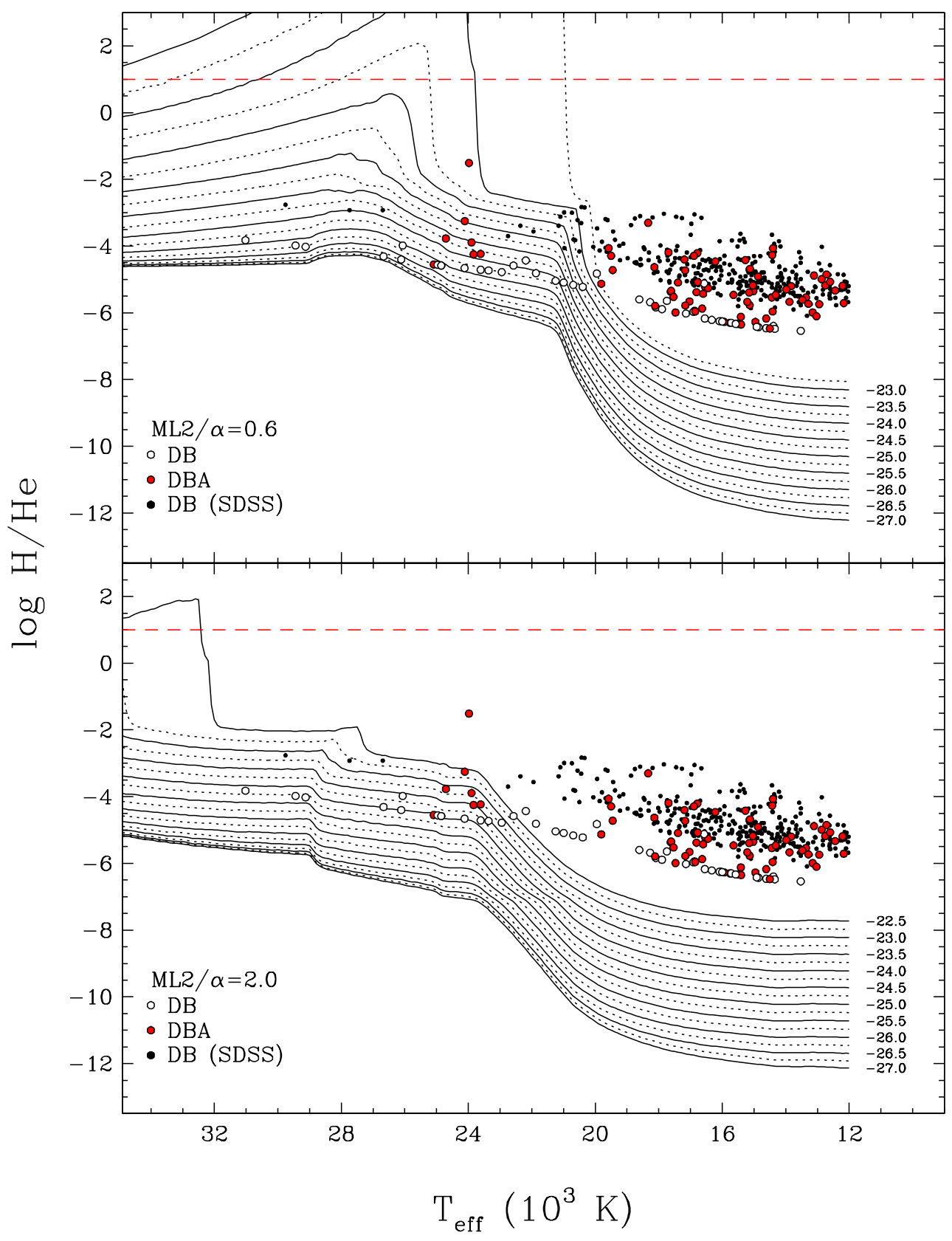

Fig. 8.- Same as Figure 7, but for a $0.6 M_{\odot}$ pure helium envelope white dwarf subject to accretion. Each curve is labeled with the corresponding accretion rate in solar mass per year (on a logarithmic scale). 


\section{A CONVECTIVE DREDGE-UP MODEL}

\subsection{The Hydrogen Diffusion Tail}

As part of an independent investigation aimed at studying the spectral evolution of hot white dwarfs (A. Bédard et al. 2020, in preparation), we performed time-dependent diffusion calculations (as opposed to static models), with a particular interest in following the diffusion of hydrogen towards the surface - the so-called float-up model — the mechanism believed to be responsible for turning most hot, helium-atmosphere white dwarfs into DA stars by the time they reach the blue (hot) edge of the DB gap (Fontaine \& Wesemael 1987). These time-dependent diffusion calculations are based on a completely new and modern implementation of an evolutionary code named STELUM - a short name for tools for STELlar modeling from Université de Montréal —, geared specifically toward stellar seismology and spectral/chemical evolution (Brassard \& Fontaine 2015). A specificity of this code is that evolutionary computations are producing complete models of stars: down from the core up to, and including, the atmosphere.

As an illustrative example, we discuss here a particular white dwarf evolutionary track at $0.6 M_{\odot}$. We begin with a typical PG1159 progenitor that has evolved through late thermal flash episodes, where the content of both hydrogen and helium has been significantly depleted (Werner \& Herwig 2006). In such a star, the stellar envelope has an extremely uniform chemical composition through intense convective mixing within the deep stellar envelope. This homogeneous chemical profile is maintained by a strong stellar wind, which eventually dies out at the end of the PG1159-phase (Quirion et al. 2012). Our initial stellar envelope is thus composed of a homogeneous mixture of $\mathrm{H} / \mathrm{He} / \mathrm{C} / \mathrm{O}$ all the way from the surface to a depth of $\log M_{\mathrm{env}} / M_{\star}=-1.87$, surrounding an inner $\mathrm{C} / \mathrm{O}$ core. The chemical composition of the atmosphere and envelope of our initial model is characterized by mass fractions of $X(\mathrm{H})=10^{-6}, X(\mathrm{He})=0.42, X(\mathrm{C})=0.43$, and $X(\mathrm{O})=0.15$, corresponding to a total amount of hydrogen of $\log M_{\mathrm{H}} / M_{\star}=-7.62\left(\right.$ or $\log M_{\mathrm{H}} / M_{\odot}=-7.84$ ), thoroughly diluted within the deep stellar envelope. Possible residual nuclear burning of hydrogen is neglected.

As time proceeds, some helium rapidly rises to the surface, transforming the PG1159 star into a DO white dwarf, and eventually into a DB white dwarf at lower effective temperatures. Hydrogen also diffuses upward, but much more slowly, eventually reaching the outer surface, at which point the object becomes a DA white dwarf. Since this is only an illustrative example, it is outside the scope of this paper to discuss all the relevant details of this particular evolutionary track. In particular, the convective dilution mechanism has not been implemented yet. Instead, we show in Figure 9 snapshots at two different epochs of the hydrogen and helium mass fractions as a function of depth. Note that these are still 
exploratory calculations, which are extremely time-consuming (several weeks of CPU time for one sequence), and we have yet to study the effect of several mechanisms, in particular convective overshooting, as discussed for instance in Cukanovaite et al. (2019). Nevertheless, our models depict the important features we want to address in this section.

In the top panel of Figure 9, we show the abundance profiles of hydrogen and helium at $T_{\text {eff }}=60,627 \mathrm{~K}$, at a point where the white dwarf still appears as a DO star, with the photospheric hydrogen abundance too low to be detected. The photospheric helium abundance is already high, however, but this is just because the initial helium content is already much larger than that of hydrogen. More of interest in the present context are the abundance profiles displayed in the bottom panel of Figure 9, at a temperature of $T_{\text {eff }}=20,247 \mathrm{~K}$, where we expect the convective dilution process to occur. Note that in this particular sequence, the amount of hydrogen accumulated in the outer envelope, $\log M_{\mathrm{H}} / M_{\star} \sim-11$, is way too large for any dilution process to occur, and this star will turn into a DC white dwarf — or a helium-rich DA star - way below $T_{\text {eff }}=10,000 \mathrm{~K}$. Nevertheless, what our calculations reveal, is that even if hydrogen is a trace element in the envelope, its abundance profile is always far from equilibrium because of the extremely large diffusion timescales in the deeper layers. In particular, near $T_{\text {eff }} \sim 20,000 \mathrm{~K}$, only $\log M_{\mathrm{H}} / M_{\star} \sim-11$ of hydrogen has accumulated in the outer layers of the white dwarf, which represents an extremely small fraction - about $0.04 \%$ - of the total hydrogen content present in the entire star $\left(\log M_{\mathrm{H}} / M_{\star}=-7.62\right)$. As a comparison, we show the hydrogen profile (in red) in full diffusive equilibrium that has a similar thickness of the outer hydrogen layer. Note that in their

calculations, MacDonald \& Vennes (1991) assumed that this full diffusive equilibrium has been reached at every $T_{\text {eff }}$ value.

The most important aspect of these calculations is that substantial amounts of hydrogen may still be located in the deeper layers of a white dwarf, regardless of the amount already accumulated at the surface. This is an unavoidable consequence due to the extremely long diffusion timescales of hydrogen in the deeper layers; note that a similar result has been obtained in the case of the helium diffusion tail by Dehner \& Kawaler (1995, see their Figure 1). Since the photosphere is usually close to the surface at these effective temperatures (see Figure 9), the majority of the hydrogen in the envelope would thus sit in a deep reservoir, inaccessible to spectroscopic observations.

\subsection{Approximate Abundance Profiles}

We now attempt to take into account the conclusions of the previous subsection summarized in Figure 9 - into our convective dilution simulations described in Section 2 . 


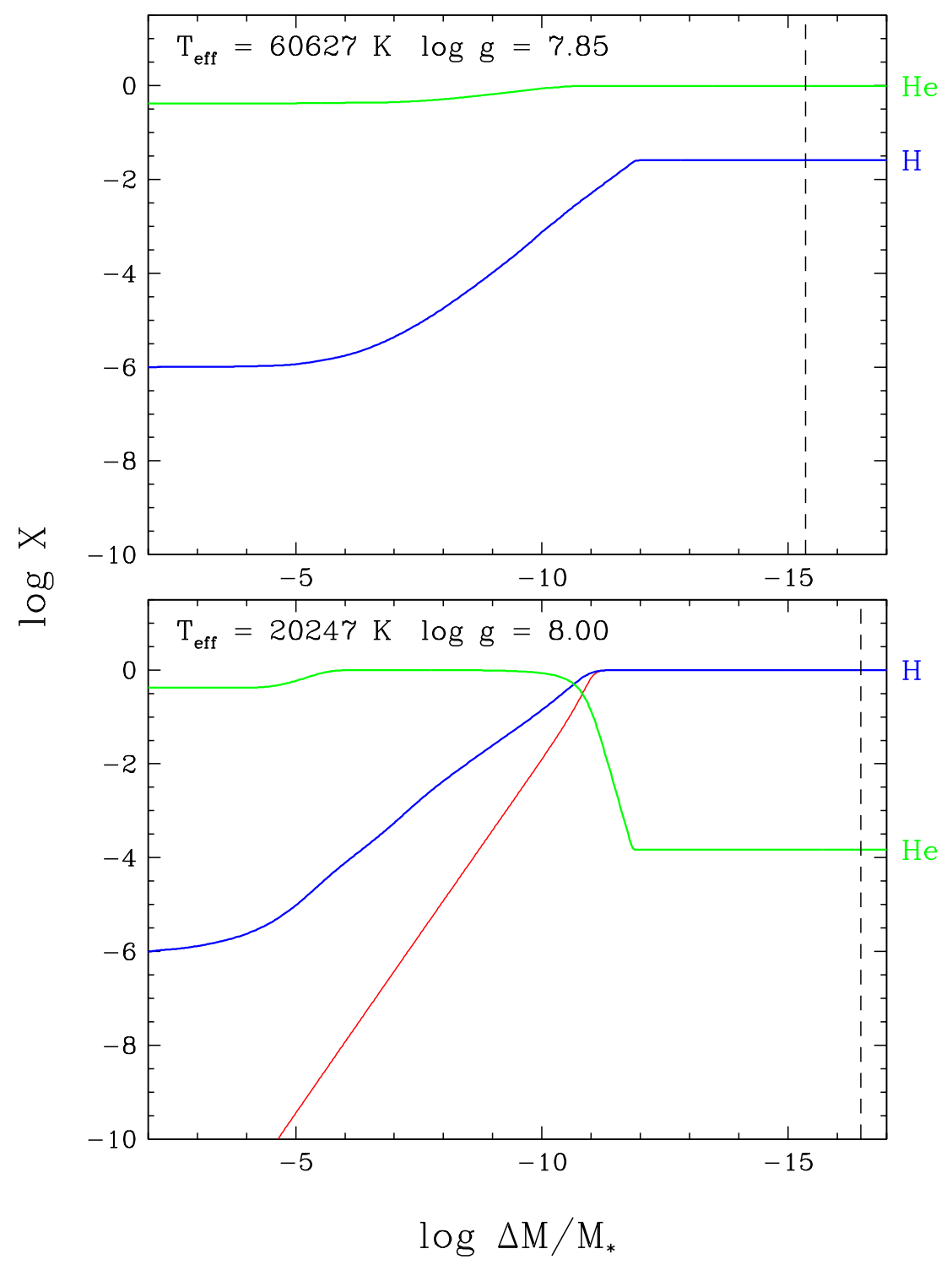

Fig. 9.- Snapshots at two different epochs of the hydrogen (blue) and helium (green) mass fractions as a function of depth, taken from time-dependent calculations of a $0.6 M_{\odot}$ white dwarf with a total hydrogen mass fraction of $X(\mathrm{H})=10^{-6}$ in the envelope. Also displayed in the bottom panel is a diffusive equilibrium profile containing $\sim 10^{-11} M_{\star}$ of hydrogen (red line). In both panels the location of the photosphere is indicated by the vertical dashed line. 
To this end, we thus consider that the overall hydrogen abundance profile can be represented by the combination of a deep reservoir and a surface contribution. Because of this massive reservoir, the superficial hydrogen layer keeps building up as a function of time (or decreasing effective temperature), but for the sake of simplicity, we assume here that the only relevant abundance profile is that when convective dilution occurs near $T_{\text {eff }} \sim 20,000 \mathrm{~K}$.

Due to the extremely large diffusion timescales found in the deeper layers, the hydrogen reservoir remains virtually unaffected in the earlier white dwarf phases, and only a small fraction of the total hydrogen mass has reached the surface at the temperature where the convective dilution process takes place $(0.04 \%$ in the example above). We therefore assume that the hydrogen mass fraction is nearly constant for $\log \Delta M / M_{\star} \lesssim-4$. In the outer layers, however, the abundance profile will, by comparison, reach its equilibrium value more quickly than in the deeper regions. For the surface contribution, we thus rely on full diffusive equilibrium profiles similar to those already described in Section 2.1 (see also MacDonald \& Vennes 1991 and Manseau et al. 2016). Below this superficial hydrogen layer of mass $M_{\mathrm{H}}$, we use two simple power laws (with exponents $-9 / 10$ and $-3 / 4$ ) to describe the hydrogen tail displayed in Figure 9, In the deeper regions, we then connect this tail to the massive reservoir at $\log \Delta M / M_{\star} \sim-4$. This connection defines the hydrogen mass fraction in the deeper envelope, and by definition the total initial mass fraction of the star. Note that the exact value is not important in the present context since the bottom of the $\mathrm{H} / \mathrm{He}$ convection zone never reaches these deeper regions.

Examples of these approximate hydrogen abundance profiles are displayed in Figure 10 for various values of the hydrogen surface layer mass, $M_{\mathrm{H}}$. For comparison, we also reproduced the detailed profile from Figure 9, which is perfectly represented by our approximate

profile with $\log M_{\mathrm{H}} / M_{\odot}=-10.35$. We can also see that with decreasing value of $M_{\mathrm{H}}$, the hydrogen mass fraction in the deeper layers decreases as well, as expected. We acknowledge that these profiles represent only an approximation of the true profiles, but we are confident that our approach represents a good characterization of the fully time-dependent, evolutionary calculations, and that we can capture the essential information required to revise the spectral evolution of DBA white dwarfs.

\subsection{Convective Dredge-up Simulations}

In order to follow the spectral evolution of white dwarfs subject to convective dredge-up, we first assume that all of the hydrogen belonging to the surface contribution (as described above) has reached the surface and obeys our new stratified envelope structures. With this approximation, we only need to take into account the contribution from the deep hydrogen 


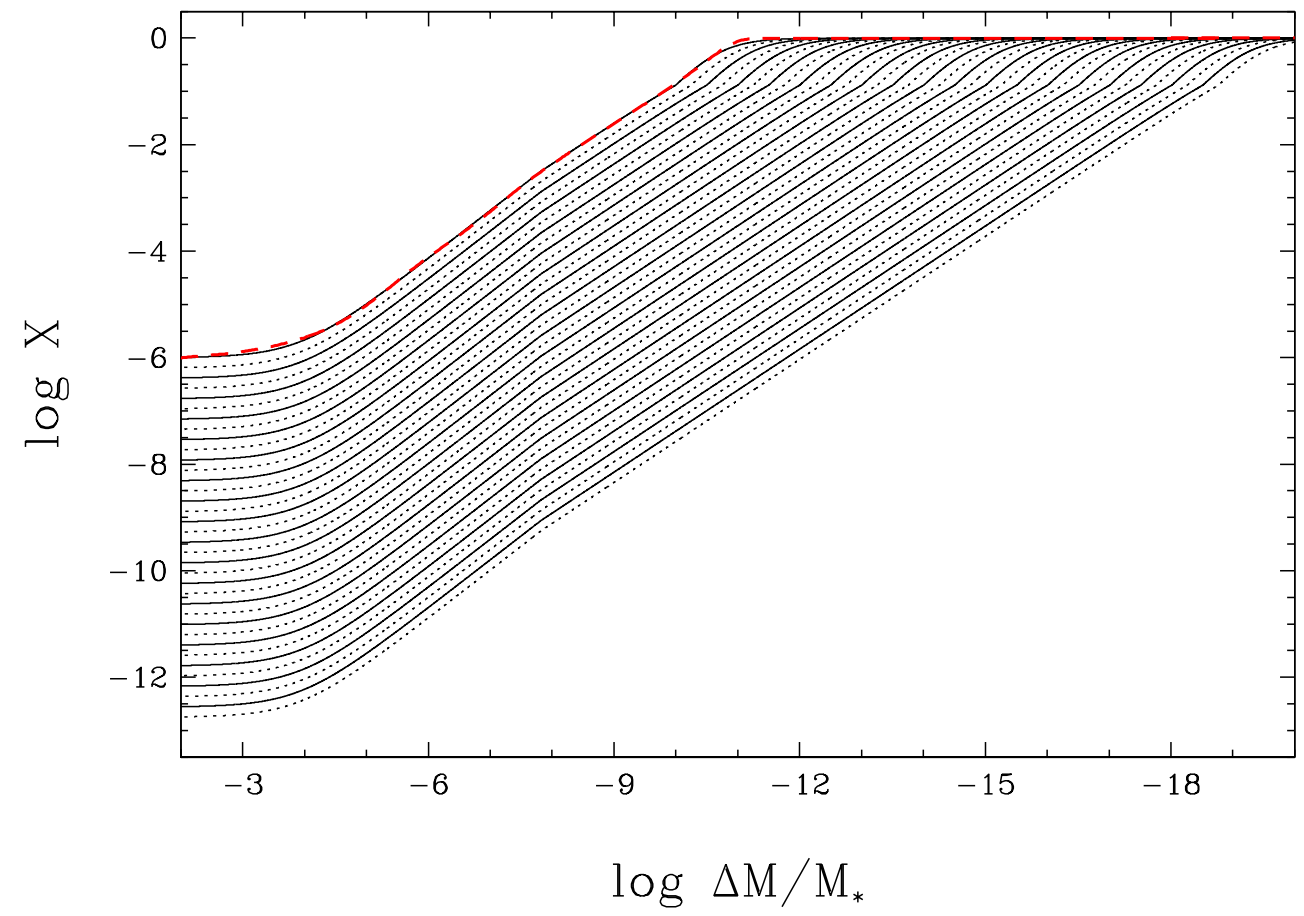

Fig. 10.- Hydrogen mass fraction as function of depth for our approximate abundance profiles in a $0.6 M_{\odot}$ white dwarf. The thickness of the hydrogen surface layer ranges from $\log M_{\mathrm{H}} / M_{\odot}=-19.0$ to -10.5 by steps of $0.25 \mathrm{dex}$ (from right to left). The detailed hydrogen profile, reproduced from Figure 9, is represented by the red dashed line together with our profile for $\log M_{\mathrm{H}} / M_{\odot}=-10.35$. 
reservoir. We then follow the same procedure outlined in Section 2, but the target value of $M_{\mathrm{H}}$ is updated at every temperature step by adding the mass of hydrogen dredged-up by the growing $\mathrm{H} / \mathrm{He}$ convection zone. Since the reservoir contains only trace amounts of hydrogen, the extent of the convection zone will not be affected (see RBF18), and the transition temperatures that take into account the convective dredge-up process are virtually identical to those given in Table 1. In other words, the convective dilution process depends on the amount of hydrogen accumulated at the surface, but it is not affected by the presence of the deep hydrogen reservoir. However, the resulting hydrogen abundances at the photosphere, after mixing has occurred, should be significantly different. We now test this new paradigm by comparing the predictions of our convective dredge-up simulations with the photospheric $\mathrm{H} / \mathrm{He}$ abundances measured in DB and DBA white dwarfs.

The results of our simulations are presented in Figure11, which can be compared directly with Figure 7, where the dredge-up process has been ignored. Our results clearly demonstrate that the addition of the deep hydrogen reservoir represents the key element to reproduce the observed hydrogen abundances in the bulk of DBA white dwarfs. The $\mathrm{H} / \mathrm{He}$ abundance ratio at $T_{\text {eff }}=15,000 \mathrm{~K}$ is predicted $\sim 2.3$ dex larger at the photosphere by including the dredge-up process. With this scenario, a significant fraction of all DBA white dwarfs can be explained with an initial superficial hydrogen layer mass lower than $\log M_{\mathrm{H}} / M_{\odot} \sim-14$, combined with a progressive enrichment from the deeper envelope layers. At the same time, the hottest DBA stars can still be explained by surface layers thinner than $\log M_{\mathrm{H}} / M_{\odot} \sim$ -16.5 , but the predicted hydrogen abundances at lower temperatures would probably be undetectable, even within the context of the dredge-up scenario. We note that some of the DBA white dwarfs in Figure 11 would require even larger hydrogen abundances, but we believe that such large abundances could probably be produced by using more accurate hydrogen abundance profiles than those displayed in Figure 10, and more importantly, by including some convective overshooting at the bottom of the mixed $\mathrm{H} / \mathrm{He}$ convection zone

(see for instance the important work by Cunningham et al. 2019 and Cukanovaite et al. 2019).

\section{DISCUSSION}

We presented a series of improved envelope models, both with stratified and mixed chemical compositions, with the aim of simulating the convective dilution process, where a thin radiative superficial hydrogen layer is gradually eroded by the deeper helium convection zone. This process is believed to be responsible for the DA-to-DB transformation below the red edge of the DB gap near $T_{\text {eff }} \sim 30,000 \mathrm{~K}$, although our calculations reveal that 


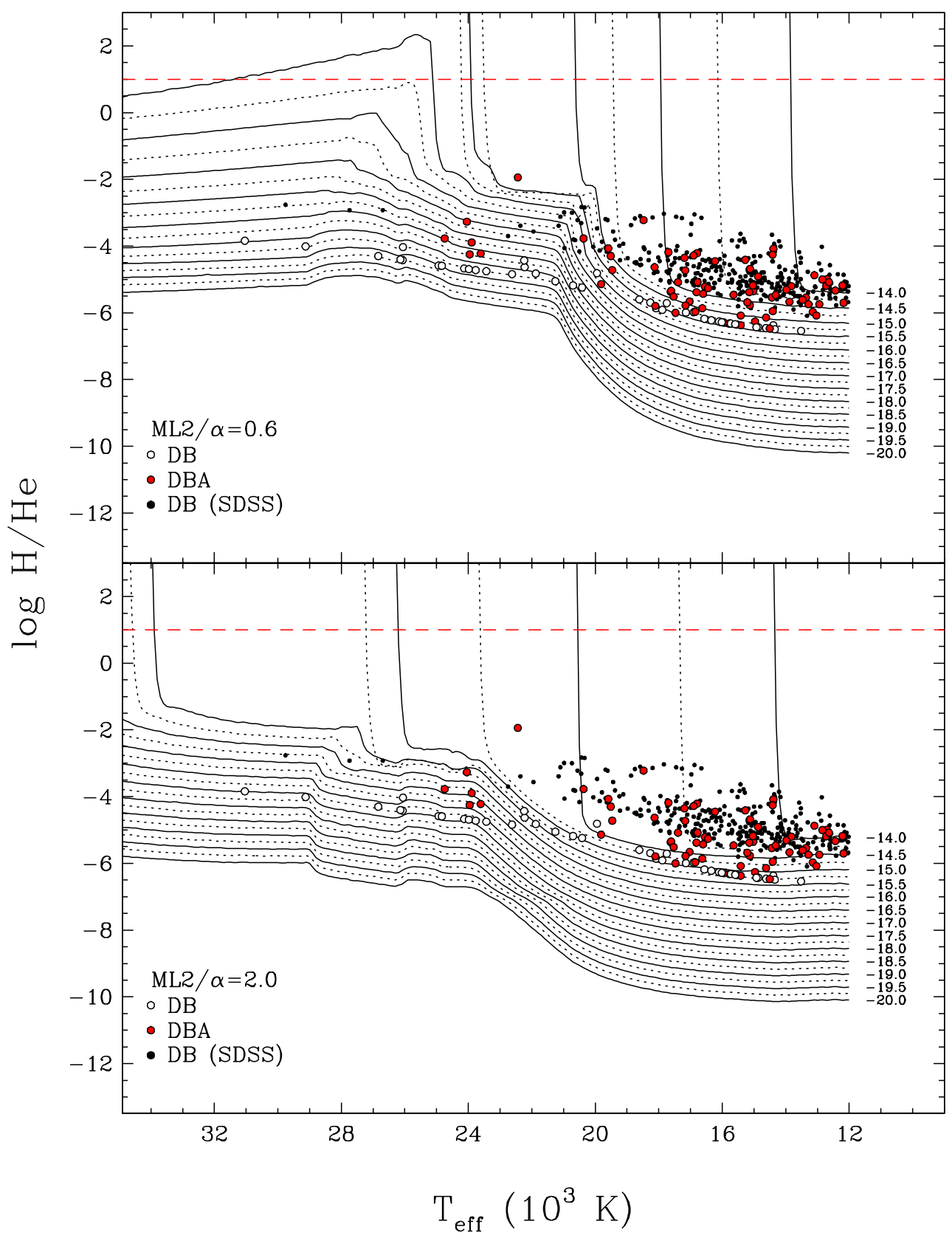

Fig. 11.- Same as Figure 7, but for a $0.6 M_{\odot}$ white dwarf undergoing convective dredge-up. Each curve is labeled with the corresponding initial hydrogen mass at the surface in solar mass (on a logarithmic scale). 
the convective dilution mechanism becomes efficient only below $T_{\text {eff }} \sim 20,000 \mathrm{~K}$, when the bottom of the helium convective envelope plunges deep into the stellar interior. If the mass of the outer hydrogen layer is too large $\left(\log M_{\mathrm{H}} / M_{\odot} \gtrsim-14\right)$, however, convective energy transport in the underlying helium envelope is suppressed, thus preventing this convection dilution process to occur. Even though the actual mixing process is obviously a complicated, time-dependent dynamical process, we believe that our static envelope models provide a fairly accurate description of the DA-to-DB transformation, in particular in terms of the effective temperatures at which this process occurs as a function of the hydrogen layer mass, as given in Table 1 .

We note that our transition temperatures differ slightly from those provided in Table 1 of MacDonald \& Vennes (1991), probably because of significant differences between their approach and ours, the most important of which is the distribution of the total hydrogen mass within the stellar envelope. In our calculations, hydrogen is distributed entirely within the mixed $\mathrm{H} / \mathrm{He}$ convection zone as well as at the stellar surface where it is assumed to be in diffusive equilibrium. More importantly, there is no hydrogen below the convection zone (note that we refer here to our calculations of the convective dilution process described in Section 2.3. where there is no deep hydrogen reservoir). In MacDonald \& Vennes, there is also a deep hydrogen diffusion tail underneath the mixed $\mathrm{H} / \mathrm{He}$ convection zone (see their Figure 6), which sometimes may involve most of the total hydrogen content. In their calculations, the authors indeed assume that a full equilibrium has been reached at every $T_{\text {eff }}$ value, allowing any amount of hydrogen present in the star to achieve a diffusive equilibrium, even below the convection zone. Hence at a given effective temperature and hydrogen mass fraction in the convection zone, the total integrated hydrogen mass in our model is much smaller than in their model, thus explaining the differences between the results in our Table 1 and their Table 1.

This being said, we believe our approach is probably more realistic in the context of the convective dilution process. Let us assume for instance that a hot DA white dwarf with a fully radiative hydrogen layer of $\log M_{\mathrm{H}} / M_{\odot}=-15$ sits in diffusive equilibrium on top of a helium envelope. When helium becomes convective, some of the hydrogen layer will be mixed within the convection zone, and some of it might leak at the bottom of the convective envelope, but certainly not to the point of achieving a full diffusive equilibrium profile. Hence it is probably better in the present context to assume that there is no hydrogen below the convection zone, at least when determining the DA-to-DB transition temperatures.

Although the exact amount of hydrogen present in the outer layers of the DA progenitor determines the temperature at which the convective dilution process will occur, the resulting hydrogen-to-helium abundance ratio measured at the photosphere of DBA white dwarfs may 
have little to do with this residual mass of hydrogen, in particular if there is a massive reservoir of hydrogen present in the deep stellar interior, which can be dredged-up to the surface by the mixed $\mathrm{H} / \mathrm{He}$ convective envelope. This is the new paradigm we explored quantitatively in Section 4 .

The overall spectral evolution picture that emerges from these calculations is the following. As discussed above, we begin with a typical PG1159 progenitor that has evolved through late thermal flash episodes (Werner \& Herwig 2006). In such a star, the content of both hydrogen and helium has been significantly depleted, and the stellar envelope has an extremely uniform chemical composition through intense convective mixing within the deep stellar envelope. The exact amount of hydrogen and helium thoroughly diluted within the deep stellar envelope is unknown, but might be extremely small. During the earlier phases, this homogeneous chemical profile is maintained by a strong stellar wind, which eventually dies out at the end of the PG1159-phase (Quirion et al. 2012). As the star cools off, helium begins to diffuse upward, thus transforming the PG1159 progenitor into a DO white dwarf. Eventually, hydrogen will also reach the surface, producing DAO white dwarfs, which will in time turn into chemically stratified DAO or DAB white dwarfs, and eventually into DA stars depending on the amount of hydrogen that has accumulated at the surface (see, e.g., Manseau et al. 2016). The important point in this scenario is that the amount of hydrogen that has reached the surface at a given point in time - i.e., at a given effective temperature - may represent only a small fraction of the total mass present in the stellar envelope. Most importantly, the hydrogen abundance profile remains always far from equilibrium because of the extremely large diffusion timescales of hydrogen in the deeper layers.

If the amount of hydrogen accumulated at the surface of the white dwarf is small enough - $\log M_{\mathrm{H}} / M_{\odot} \lesssim-14$ according to our results given in Table 1 - the convective dilution of this thin hydrogen layer by the more massive underlying convective helium envelope will occur, at a mixing temperature that depends on the hydrogen layer mass. At this point, the bottom of the mixed $\mathrm{H} / \mathrm{He}$ convective reaches deep into the stellar interior, where large amounts of hydrogen may still reside. The convective dredge-up process that follows may potentially carry large amounts of hydrogen to the stellar photosphere, depending on the exact quantity of hydrogen present in the deeper interior. In this scenario, the amount of hydrogen originally present at the surface, prior to the convective dilution process, represents only a negligible fraction of the total mass of hydrogen present in the mixed $\mathrm{H} / \mathrm{He}$ convective envelope after the dredge-up process has occurred.

On the other hand, if the amount of hydrogen accumulated at the surface of the white dwarf progenitor is too small - $\log M_{\mathrm{H}} / M_{\odot} \lesssim-16$ according to Manseau et al. (2016) the star will never become a genuine DA white dwarf, and will only appear as a stratified 
DAB star, as discussed in Section 2.3. In this context, we interpreted the hot DBA white dwarfs near $T_{\text {eff }} \sim 24,000 \mathrm{~K}$ in Figure 7 as descendants of such hydrogen-poor progenitors. We also mentioned that with time, these DBA white dwarfs would eventually turn into DB stars, with no detectable traces of hydrogen, when the growth of the mixed $\mathrm{H} / \mathrm{He}$ convective zone below $T_{\text {eff }} \sim 20,000 \mathrm{~K}$ completely dilutes any amount of hydrogen present in the outer layers. However, within the context of the convective dilution scenario, it is also possible that the growth of the convection zone is accompanied by a hydrogen enrichment from the deeper layers into the photospheric regions, in which case the star could remain a DBA white dwarf.

If the convective dilution scenario is the correct explanation for the observed abundances of hydrogen in the bulk of DBA white dwarfs, we are forced to conclude that the pure DB stars below $T_{\text {eff }}=20,000 \mathrm{~K}$ - those that show no $\mathrm{H} \alpha$ absorption feature whatsoever - must have very little hydrogen left in their stellar envelope, even in the deeper regions, most likely because of very intense and repeated late thermal flash episodes in their earlier evolutionary phases. If this is indeed the case, these hydrogen-free DB stars could represent the progenitors of the cool DQ white dwarfs, in which hydrogen is rarely detected spectroscopically, as opposed to other cool, non-DA degenerates (the DZA stars in particular).

Finally, it is clear that accretion of hydrogen from external sources - mostly from comets, disrupted asteroids, and small planets - play an important role in the spectral evolution of helium-atmosphere white dwarfs. In fact, the extreme hydrogen and metal abundances measured in some of these objects can only be explained by such accretion mechanisms. However, as discussed in Section 3, accretion needs to proceed after the convective dilution process has occurred, otherwise, even modest average accretion rates would build over time a hydrogen layer so thick that the DA-to-DB transition becomes impossible. For these reasons, we believe that the convective dredge-up model, proposed in this paper to explain the origin of hydrogen in the bulk of DBA white dwarfs, represents a more plausible scenario. One could even argue that, if the overall picture described above is correct, hydrogen enrichment due to convective dredge-up is an unavoidable outcome. Additional time-dependent calculations such as those illustrated in Figure 9 should eventually shed some light on this issue.

We are grateful to C. Genest-Beaulieu and A. Bédard for useful discussions. This work was supported in part by the NSERC Canada and by the Fund FRQ-NT (Québec). G.F. also acknowledges the contribution of the Canada Research Chair Program. 


\section{REFERENCES}

Bergeron, P., Wesemael, F., Dufour, P., Beauchamp, A., Hunter, C., Saffer, R. A., Gianninas, A., Ruiz, M. T., Limoges, M.-M., Dufour, P., Fontaine, G., \& Liebert, J. 2011, ApJ, 737,28

Brassard, P. \& Fontaine, G. 1994, in IAU Colloq. 147: The Equation of State in Astrophysics, ed. G. Chabrier \& E. Schatzman, Vol. 147, 560

Brassard, P. \& Fontaine, G. 2015, in Astronomical Society of the Pacific Conference Series, Vol. 493, 19th European Workshop on White Dwarfs, ed. P. Dufour, P. Bergeron, \& G. Fontaine, 125

Cukanovaite, E., Tremblay, P. E., Freytag, B., Ludwig, H. G., Fontaine, G., Brassard, P., Toloza, O., \& Koester, D. 2019, MNRAS, 490, 1010

Cunningham, T., Tremblay, P.-E., Freytag, B., Ludwig, H.-G., \& Koester, D. 2019, MNRAS, 488, 2503

Dantona, F. \& Mazzitelli, I. 1979, A\&A, 74, 161

Dehner, B. T. \& Kawaler, S. D. 1995, ApJ, 445, L141

Eisenstein, D. J., Liebert, J., Koester, D., Kleinmann, S. J., Nitta, A., Smith, P. S., Barentine, J. C., Brewington, H. J., Brinkmann, J., Harvanek, M., Krzesiński, J., Neilsen, Jr., E. H., Long, D., Schneider, D. P., \& Snedden, S. A. 2006, AJ, 132, 676

Farihi, J., Gänsicke, B. T., \& Koester, D. 2013, Science, 342, 218

Fontaine, G., Brassard, P., \& Bergeron, P. 2001, PASP, 113, 409

Fontaine, G. \& Wesemael, F. 1987, in IAU Colloq. 95: Second Conference on Faint Blue Stars, ed. A. G. D. Philip, D. S. Hayes, \& J. W. Liebert, 319-326

Gentile Fusillo, N. P., Gänsicke, B. T., Farihi, J., Koester, D., Schreiber, M. R., \& Pala, A. F. 2017, MNRAS, 468, 971

Green, R. F., Schmidt, M., \& Liebert, J. 1986, ApJS, 61, 305

Greenstein, J. L. 1986, ApJ, 304, 334

Jura, M. 2003, ApJ, 584, L91

Koester, D. 1976, A\&A, 52, 415 
Koester, D. \& Kepler, S. O. 2015, A\&A, 583, A86

Liebert, J., Wesemael, F., Hansen, C. J., Fontaine, G., Shipman, H. L., Sion, E. M., Winget, D. E., \& Green, R. F. 1986, ApJ, 309, 241

Limoges, M.-M., Bergeron, P., \& Lépine, S. 2015, ApJS, 219, 19

MacDonald, J. \& Vennes, S. 1991, ApJ, 371, 719

Manseau, P. M., Bergeron, P., \& Green, E. M. 2016, ApJ, 833, 127

Paquette, C., Pelletier, C., Fontaine, G., \& Michaud, G. 1986, ApJS, 61, 197

Quirion, P.-O., Fontaine, G., \& Brassard, P. 2012, ApJ, 755, 128

Raddi, R., Gänsicke, B. T., Koester, D., Farihi, J., Hermes, J. J., Scaringi, S., Breedt, E., \& Girven, J. 2015, MNRAS, 450, 2083

Rolland, B., Bergeron, P., \& Fontaine, G. 2018, ApJ, 857, 56

Schatzman, E. L. 1958, White dwarfs

Tremblay, P.-E. \& Bergeron, P. 2008, ApJ, 672, 1144

Van Grootel, V., Charpinet, S., Brassard, P., Fontaine, G., \& Green, E. M. 2013, A\&A, 553, A97

Vauclair, G. \& Reisse, C. 1977, A\&A, 61, 415

Vennes, S., Pelletier, C., Fontaine, G., \& Wesemael, F. 1988, ApJ, 331, 876

Veras, D., Shannon, A., \& Gänsicke, B. T. 2014, MNRAS, 445, 4175

Werner, K. \& Herwig, F. 2006, PASP, 118, 183

Wesemael, F., Greenstein, J. L., Liebert, J., Lamontagne, R., Fontaine, G., Bergeron, P., \& Glaspey, J. W. 1993, PASP, 105, 761 